\title{
Un modèle pour l'étude de la transmission de l'information produite par certains facteurs de croissance : les mécanismes d'activation de la S6 kinase dans les astrocytes en culture
}

\author{
D. Toru-Delbauffe, J.M. Gavaret, C. Matricon, M. Pomerance, \\ C. Jacquemin et M. Pierre
}

INSERM U. 96, unité de recherche sur la glande thyroïde et la régulation hormonale, 78 , rue du Général-Leclerc, 94275 Le Kremlin-Bicêtre Cedex, France

(14 Réunion du groupe Développement INRA, Clermont-Ferrand, 25-27 mai 1988)

\begin{abstract}
Résumé - Le travail a été effectué sur des cultures d'astrocytes provenant d'hémisphères cérébraux de rats nouveau-nés de 2 jours. Le traitement de ces cellules par l'insuline, la somatomédine C (IGF1), la thrombine et les facteurs de croissance acide et basique des fibroblastes (FGFs) provoque une rapide activation de la protéine kinase cytosolique ( 66 kinase), qui phosphoryle la protéine ribosomale S6. L'ester de phorbol (TPA) produit aussi une rapide augmentation de l'activité de cette enzyme. Deux agonistes de l'adénylate cyclase (forskoline et isoprotérénol) ainsi que l'analogue de I'AMP cyclique (dibutyryl AMPc) stimulent, eux aussi, cette enzyme. Ces observations suggèrent la possiblité que la même $\mathbf{S} 6$ kinase, considérée comme l'une des premières cibles des signaux produits par les facteurs de croissance, serait activée par des voies distinctes.
\end{abstract}

astrocytes - facteurs de croissance — agents mitogènes — phosphorylation — protéine S6

Summary - A model for studying transmission of information elicited by certain growth factors : S6 kinase activation mechanisms in cultured astrocytes. Treatment of cultured astrocytes from 2-day-old rat cerebral hemispheres with insulin, somatomedin C (IGF1), thrombin and acidic or basic fibroblast growth factors promoted a rapid activation of a cytosolic protein kinase (S6 kinase) which phosphorylates ribosomal protein S6. The phorbol ester (TPA) also triggered a rapid increase in S6 kinase activity. Two agonists of adenylate cyclase activity (forskolin and isoproterenol) and the cyclic AMP analog (dibutyryl cAMP) also stimulated the same S6 kinase. These observations support the idea that several pathways might promote the activation of the same entity that is regarded as one of the primary targets of signals elicited by growth factors.

astrocytes - growth factors - mitogen agents - phosphorylation — S6 kinase

\section{Abréviations}

EGF : epidermal growth factor; IGF1 : insulin-like growth factor; FGFa : acidic fibroblast growth factor; FGFb : basic fibroblast growth factor; PDGF : platelet-derived growth factor; PGF2 $\alpha$ : prostaglandin F2 $\alpha$; PL : phospholipides; TPA : 12-0-tétradécanoylphorbol-13-acétate; DMEM : Dulbecco's modified Eagle's medium; PAK II : protease-activated kinase II; H4P kinase: H4-specific protein kinase; DTT : dithiothréitol; NEM : N-éthylmaléimide. 


\section{INTRODUCTION}

Lorsque des cellules quiescentes en culture sont stimulées par du sérum ou des facteurs de croissance individuels, un certain nombre de processus biologiques sont déclenchés. Ils aboutissent à la prolifération cellulaire. En dépit de l'importance fondamentale de ce mécanisme, les bases de son contrôle moléculaire ne sont pas encore bien établies.

Pour comprendre comment la prolifération est régulée, il est nécessaire de déterminer les étapes qui permettent à un signal extracellulaire de provoquer l'initiation de la synthèse de l'ADN, et par conséquent la division cellulaire. La synthèse protéique est une des étapes dont le rôle est central. Thomas et al. (1979) ont constaté que l'addition de sérum à des fibroblastes quiescents 3T3 se traduit par une accumulation accrue de certaines protéines. Cet événement est en corrélation avec une augmentation de vitesse de l'initiation de la synthèse protéique et avec une augmentation de phosphorylation de la protéine $S 6$ de la sous-unité ribosomale 40S. La phosphorylation de cette protéine provoquerait une meilleure efficacité de traduction de certains ARN messagers. En effet, Palen \& Traugh, en 1987, ont montré in vitro que l'augmentation de synthèse de certaines protéines pouvait être due à la phosphorylation de la protéine S6 qui peut modifier la traduction de leur ARN messager. Depuis quelques années, il a été constaté que de nombreux facteurs mitogènes ( $E G F$, insuline et IGF1, FGFa et FGFb, PDGF, PGF2 $\alpha$ et esters de phorbol), ainsi que certains virus oncogènes codant pour une tyrosine kinase (TK), produisent une multiple phosphorylation de la protéine S6. Cette phosphorylation a donc été prise comme indice par plusieurs équipes, dont la nôtre, pour étudier la transmission de l'information des agents mitogènes dans le cytosol, d'autant qu'elle est un signal précoce, produit quelques minutes après la fixation des agents mitogènes sur leurs récepteurs membranaires.

Les agents mitogènes cités précédemment induisent la phosphorylation de la protéine S6 par des voies différentes. En effet, on sait que certains activent une tyrosine kinase membranaire, d'autres la protéine kinase stimulée par les phospholipides (PKC). Enfin, le mode d'action de certains autres est encore inconnu.

Depuis quelques années, des enzymes phosphorylant la protéine S6 et dont l'activité est stimulée par des facteurs de croissance ou des virus oncogènes codant pour une tyrosine kinase ont été identifiées dans plusieurs types cellulaires : lignées de fibroblastes (Novak-Hofer \& Thomas, 1984; Blenis et al., 1984; Tabarini et al., 1985; Trevillyan et al., 1985; Cobb, 1986; Hecht \& Straus, 1986; Pelech et al., 1986; Jakubowicz \& Leader, 1987), de PC 12 (Matsuda et al., 1986), de lymphocytes (Evans \& Farrar, 1987), fibroblastes d'embryon de poulet (Blenis et al., 1984), astrocytes (Pierre et al., 1986), œufs ou oocytes de Xénope (Erikson \& Maller, 1985; Stefanovic et al., 1986) et d'étoile de mer (Pelech et al., 1987a). Ces enzymes ont pu être distinguées d'autres activités phosphorylant la protéine S6 in vitro [protéines kinases dépendantes de l'AMP cyclique, du GMP cyclique, protéine kinase $C$, protéine kinase $M$, protéine kinase dépendante de la calmoduline, S6 kinases activées par protéolyse : PAK II (Perisic \& Traugh, 1985) et H4P kinase (Donahue \& Masaracchia, 1984)].

\section{MATÉRIEL ET MÉTHODES}

Pour l'étude de la multiplication et de la différenciation des cellules du système ner- 
veux central, nous avons choisi le modèle des astrocytes. Dans ce système, une activité S6 kinase activée par l'insuline a été mise en évidence (Pierre et al., 1986). Comme les astrocytes répondent tant par leur croissance que par leur différenciation à l'IGF1, à l'insuline, aux FGFs, à la throm- bine et au TPA ainsi qu'aux agents qui augmentent le taux intracellulaire d'AMP cyclique, nous avons étudié la régulation éventuelle de la S6 kinase par ces divers facteurs selon le protocole résumé dans le Tableau I.

Tableau I. Préparation du cytosol et principe du dosage de l'activité S6 K (Pierre et al., 1986).

Cellules enrichies en astrocytes préparées à partir d'hémisphères cérébraux de rats de 2 jours

Cultivées $1-2$ semaines en DMEM + 10\% sérum veau fœtal

\section{Confluence}

$\checkmark$

Milieu chimiquement défini (Bottenstein \& Sato, 1979)

8 jours

Carence en insuline $48 \mathrm{~h}$ avant traitement par facteurs de croissance $(0 \rightarrow 1 \mathrm{~h})$

Lavage des cellules : tampon A

- $80 \mathrm{mmol} \beta$ glycérophosphate pH 7,4

- $20 \mathrm{mmol}$ EGTA

- $15 \mathrm{mmol} \mathrm{MgCl} 2$

Grattage des cellules dans le tampon A + inhibiteurs de protéases

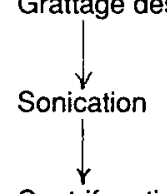

Centrifugation $100000 \mathrm{~g}(1 \mathrm{~h})$

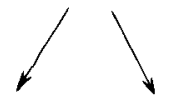

Culot cellulaire éliminé
Surnageant
- Mesure de l'activité S6 K de l'extrait total avec ${ }^{32} \mathrm{P}$ ATP $+40 \mathrm{~S}$ de ribosomes de foie. La protéine $S 6$ marquée au ${ }^{32} P$

est repérée sur gel de polyacrylamide + SDS.

La bande est découpée et sa radioactivité est comptée.

- Purification de la S6 kinase, afin de comparer les activités stimulées par les différents facteurs. 


\section{RÉSULTATS ET DISCUSSION}

L'incubation des cultures d'astrocytes pendant $1 \mathrm{~h}$ avec les agents mitogènes suivants : insuline et IGF1, thrombine, TPA et FGFs, utilisés à la concentration qui donne la stimulation maximale de l'activité S6 kinase, permet de constater que le TPA est le meilleur effecteur (Pierre et al., 1986) et que le FGFa est aussi actif que le FGFb. Cette similitude dans la stimulation de l'activité S6 kinase par les 2 FGFs contraste avec leur différence d'efficacité sur la prolifération de fibroblastes, qui est prise comme critère pour la mesure de leur activité (Gavaret et al., 1989).

L'importance de la stimulation de l'activité S6 kinase par le TPA nous a incité à choisir le traitement des astrocytes par ce facteur pour entreprendre l'analyse de(s) enzyme(s) responsable(s) de cette activité. Tout d'abord il est apparu qu'une seule enzyme catalyse la phosphorylation de la protéine S6 après traitement par le TPA. En effet, l'activité est éluée en un seul pic après chromatographies successives sur DEAE Sephacel et phosphocellulose, suivies d'une centrifugation en gradient de saccharose (Toru-Delbauffe et al., 1988). Cette enzyme est distincte de la protéine kinase $C$ qui disparaît du cytosol par translocation après traitement des astrocytes pendant $1 \mathrm{~h}$ par le TPA (Pierre et al., 1986), alors que de manière concomitante l'activité S6 kinase augmente. II est aussi séparé de la protéine kinase $A$ (PKA) et des caséines kinases.

Quelques-unes de ses propriétés réunies dans le Tableau II ont été établies après sa purification partielle (ToruDelbauffe et al., 1988). L'identité des propriétés de cette enzyme avec celles de l'enzyme obtenue après traitement des astrocytes par le FGFa (Gavaret et al., 1989) ou l'insuline et l'IGF1 (travaux non publié) suggère que les signaux produits par ces divers stimuli convergent sur une seule S6 kinase pour l'activer.

Cette conclusion est en accord avec les résultats préliminaires de Blenis \& Erickson (1985) qui ont montré, sur des fibroblastes d'embryon de poulet, qu'une protéine kinase commune est activée par le sérum, le TPA et le produit du gène du virus de sarcome de Rous, et avec ceux pius récents de Pelech \& Krebs (1987b) qui suggèrent qu'une simple protéine kinase est stimulée par traitement des cellules 3 T3 avec PDGF, EGF et insuline.

Le groupe d'Erickson (Blenis et al., 1987 ) et celui de Thomas (Jenö et al., 1988) ont purifié à l'homogénéité la S6 kinase à partir de sources cellulaires différentes. Son poids moléculaire a été déterminé, il est de l'ordre de $75 \mathrm{kDa}$, bien que certaines incertitudes persistent. La plupart de ses propriétés coïncident avec celles énoncées pour la S6 kinase d'astrocytes, ce qui permet d'envisager que l'enzyme est ubiquitaire.

Etant donné que certains auteurs ont rapporté que l'AMP cyclique module la croissance cellulaire (Pastan et al., 1975; Rozengurt, 1980), nous avons examiné son éventuel rôle dans la voie d'activation de la S6 kinase, en traitant les astrocytes par des agents qui augmentent le niveau intracellulaire de l'AMP cyclique, comme la forskoline et l'isoprotérénol ou par un dérivé de l'AMP cyclique, le dibutyryl cAMP. Nous avons constaté que ces agents produisent une forte activation de la $56 \mathrm{ki}$ nase (Gavaret et al., 1989); il s'ensuit donc que cette enzyme est régulée par la PKA.

En conclusion, la S6 kinase, qui semble être un bon candidat parmi les facteurs possibles du contrôle de la biosynthèse des protéines, est effectivement un point de confluence de plusieurs voies de régulation. Les étapes du mécanisme molécu- 
Tableau II. Propriétés de la S6 kinase des astrocytes.

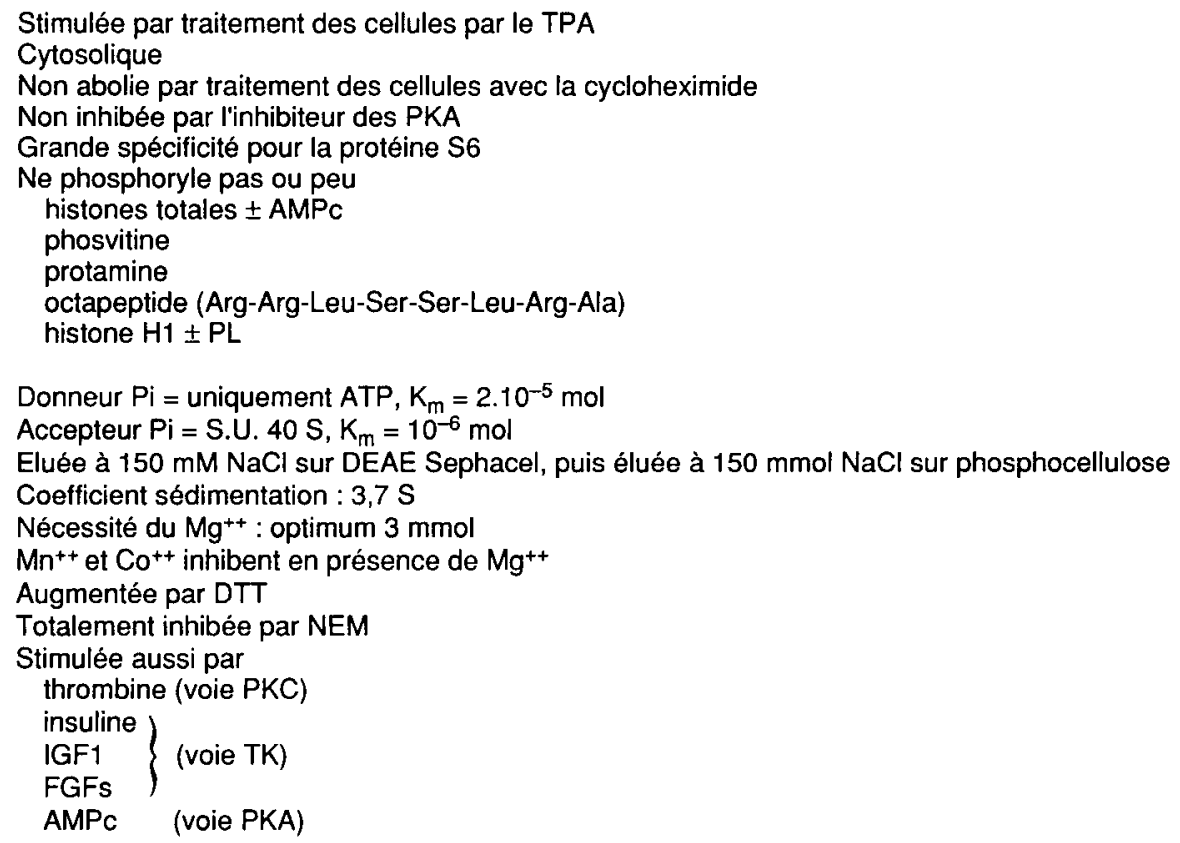

laire de cette activation sont en cours d'étude. A l'heure actuelle, certains points sont connus. En effet, Novak-Hofer \& Thomas (1985) ont montré que l'activation de la S6 kinase n'est obtenue et maintenue qu'en incluant durant la préparation des extraits cellulaires, des inhibiteurs de protéases et surtout des inhibiteurs de phosphatases. Cette observation suggère fortement que l'activation de l'enzyme procède d'un mécanisme de phosphorylationdéphosphorylation. Les travaux récents de Ballou et al. (1988) ont confirmé cette hypothèse en montrant que la protéine phosphatase 2A inhibe fortement la S6 kinase dans les fibroblastes 3T3. Mais à ce jour, l'activation de la S6 kinase par phosphorylation directe de l'enzyme n'a pu être montrée. Des auteurs suggèrent que la phos- phorylation de la S6 kinase est sous le contrôle de la protéine kinase $C$ (Pelech \& Krebs, 1987b; Evans \& Farrar, 1987) ou de la protéine kinase dépendante de l'AMP cyclique (Matsuda \& Guroff, 1987), mais la démonstration de la phosphorylation n'a pas encore été faite. Nous avons tenté d'activer la S6 kinase d'astrocytes par la sous-unité catalytique de la protéine kinase dépendante de l'AMP cyclique, mais notre essai s'est révélé infructueux.

Des résultats récents laissent pressentir que la phosphorylation de la S6 kinase ne se produit que sur des résidus sérine. Dans le cas, en particulier, des agents mitogènes qui stimulent une tyrosine kinase, il est donc nécessaire d'envisager l'existence d'intermédiaire(s) entre leurs récepteurs membranaires et la S6 kinase, pour 
expliquer leur effet sur l'enzyme. Une partie de notre travail est orientée sur la recherche de telles molécules, cibles en particulier de la tyrosine kinase du récepteur d'IGF1. Actuellement, nous procédons à la purification d'un activateur soluble de la S6 kinase et l'étude de son mécanisme d'action est en cours.

Toutes ces observations permettent d'élaborer le schéma de la Figure 1 qui pourrait expliquer les étapes du contrôle de la régulation de la S6 kinase.

\section{REMERCIEMENTS}

Nous remercions le Dr Barritault (université Paris XII) pour son don gracieux des FGFs, le Dr Lavielle (université Pierre et Marie Curie) pour son aide dans la synthèse du S6 peptide, ainsi que Mme Lefèvre et $M$. Bahloul pour la préparation de ce manuscrit. Ce travail a été subventionné par l'Association pour la Recherche contre le Cancer et l'université Paris VII.

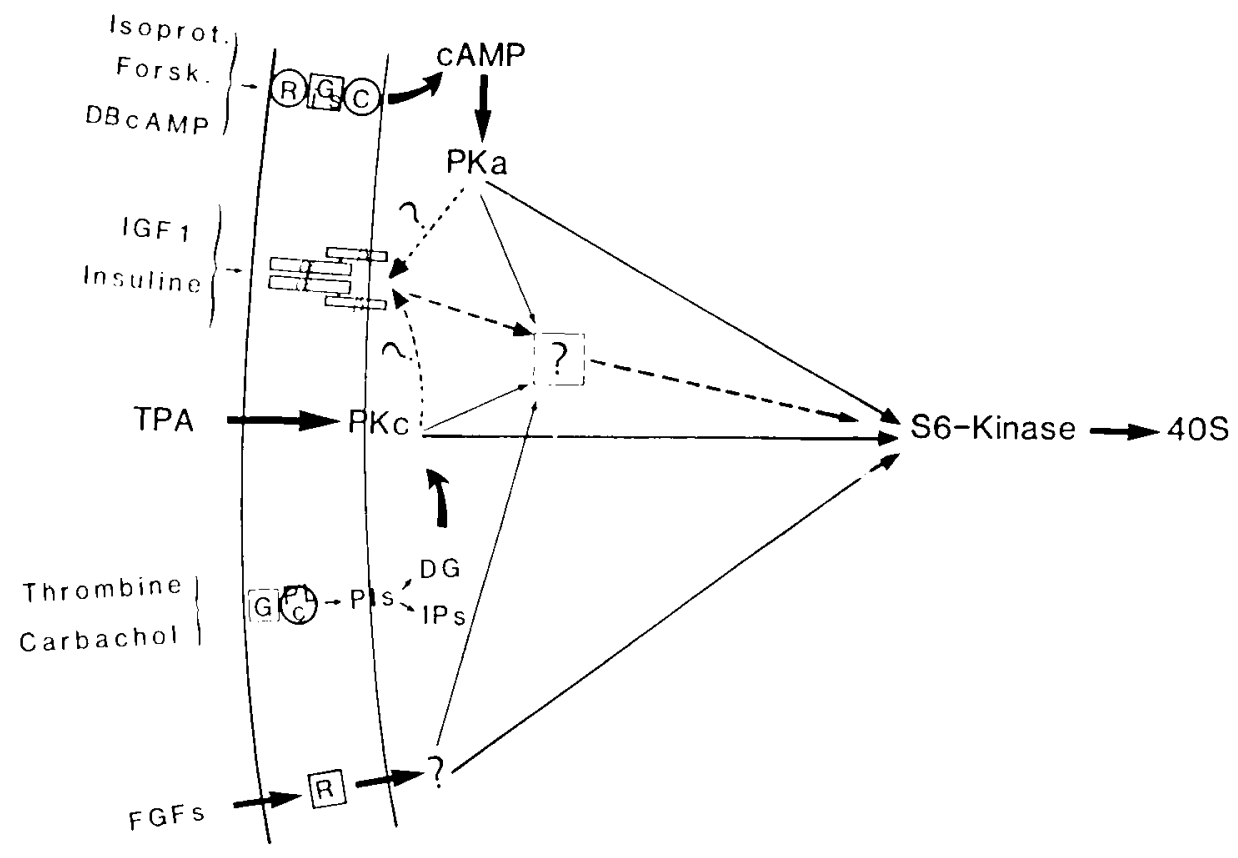

Fig. 1. Schéma possible des mécanismes de l'activation de la $S 6$ kinase. $R_{i} G_{s} C:(R)=$ sous-unité régulatrice de la PKA; $(C)=$ sous-unité catalytique de la PKA; $(G)=$ protéine $G$, inhibitrice (I) ou stimulatrice $(S) ;(\alpha \beta)_{2}$ : récepteur de l'insuline ou de l'IGF1, $\alpha=$ sous-unité de liaison du facteur, $\beta=$ sousunité catalytique (tyrosine kinase); G - PLc + PIs = DG + IPs : phospholipase C (protéine G) catalyse la transformation des phosphatidylinositols (PIs) en diacylglycérol (DG) et inositols phosphate (IPs); $R=$ récepteur non caractérisé des FGFs. 


\section{RÉFÉRENCES}

Ballou L.M., Jenö P. \& Thomas G. (1988) Protein phosphatase $2 A$ inactivates the mitogenstimulated S6 kinase from Swiss 3 T3 cells. $J$. Biol. Chem. 263, 1188-1194

Blenis J., Spivack J.G. \& Erikson R.L. (1984) Phorbol ester, serum and Rous sarcoma virus transforming gene product induce similar phosphorylations of ribosomal protein S6. Proc. Natl Acad. Sci. USA 81, 6408-6412

Blenis J. \& Erickson R.L. (1985) Regulation of a ribosomal protein $\mathrm{S} 6$ kinase activity by the Rous sarcoma virus transforming protein, serum or phorbol ester. Proc. Natl Acad. Sci. USA 82, 7621.7625

Blenis J., Kuo C.J. \& Erikson R.L. (1987) Identification of a ribosomal protein $\mathrm{S} 6$ kinase regulated by transforming and growth-promoting stimuli. J. Biol. Chem. 262, 14373-14376

Bottenstein J.E. \& Sato G.N. (1979) Growth of a rat neuroblastoma cell line in serum-free supplemented medium. Proc. Natl Acad. Sci. USA 76, 514-517

Cobb M.H. (1986) An insulin-stimulated ribosomal protein S6 kinase in 3T3-L1 cells. J. Biol. Chem. 261, 12994-12999

Donahue M.J. \& Masaracchia R.A. (1984) Phosphorylation of ribosomal protein $\mathrm{S} 6$ at multiple sites by a cyclic AMP-dependent protein kinase from lymphoid cells. J. Biol. Chem. 259, 435440

Erikson E. \& Maller J.L. (1985) A protein kinase from xenopus eggs specific for ribosomal protein S6. Proc. Natl Acad. Sci. USA 82, 742-746

Evans S.W. \& Farrar W.L. (1987) Interleukin 2 and diacylglycerol stimulate phosphorylation of 40 S ribosomal S6 protein. J. Biol. Chem. 262, 4624-4630

Gavaret J.M., Matricon C., Pomerance M., Jacquemin C., Toru-Delbauffe D. \& Pierre M. (1989) Activation of S6 kinase in astroglial cells by FGFa and FGFb. Dev. Brain Res. 45, 77-82

Hecht L.B. \& Straus D.S. (1986) Insulinsensitive, serum-sensitive protein kinase activity that phosphorylates ribosomal protein S6 in cultured fibroblast-melanoma hybrid cells. Endocrinology 119, 470-480

Jakubowicz T. \& Leader D.P. (1987) Induction, partial purification and characterization of a hamster fibroblast protein kinase activity that phosphorylates ribosomal protein S6. Eur. J. Biochem. 164, 83-88

Jenö P., Ballou L.M., Novak-Hofer I. \& Thomas G. (1988) Identification and characterization of a mitogen-activated $\mathrm{S} 6$ kinase. Proc. Natl Acad. Sci. USA 85, 406-410

Matsuda Y., Nakanishi N., Dickens G. \& Guroff G. (1986) A nerve growth factor-sensitive S6 kinase in cell-free extracts from PC 12 cells. $J$. Neurochem. 47, 1728-1734

Matsuda Y. \& Guroff G. (1987) Purification and mechanism of activation of a nerve growth factor-sensitive $\mathrm{S} 6$ kinase from $\mathrm{PC} 12$ cells. J. Biol. Chem. 262, 2832-2844

Novak-Hofer I. \& Thomas G. (1984) An activated $\mathrm{S} 6$ kinase in extracts from serum -and epidermal growth factor-stimulated Swiss 3T3 cells. J. Biol. Chem. 259, 5995-6000

Novak-Hofer I. \& Thomas G. (1985) Epidermal growth factor-mediated activation of an $\mathrm{S} 6 \mathrm{ki}-$ nase in Swiss mouse 3T3 cells. J. Biol. Chem. 260, 10314-10319

Palen E. \& Traugh J.A. (1987) Phosphorylation of ribosomal protein S6 by CAMP-dependent protein kinase and mitogen-stimulated $\mathrm{S} 6 \mathrm{ki}-$ nase differentially alters translation of globin mRNA. J. Biol. Chem. 262, 3518-3523

Pastan I.H., Johnson G.S. \& Anderson W.B. (1975) Role of cyclic nucleotides in growth control. Ann. Rev. Biochem. 44, 491-522

Pelech S.L., Olwin B.B. \& Krebs E.G. (1986) Fibroblast growth factor treatment of Swiss $3 \mathrm{~T} 3$ cells activates a subunit 56 kinase that phosphorylates a synthetic peptide substrate. Proc. Natl Acad. Sci. USA 83, 5968-5972

Pelech S.L., Meijer L. \& Krebs E.G. (1987a) Characterization of maturation-activated histone $\mathrm{H} 1$ and ribosomal S6 kinases in sea star oocytes. Biochemistry 26, 7960-7968

Pelech S.L. \& Krebs E.G. (1987b) Mitogenactivated S6 kinase is stimulated via protein kinase $\mathrm{C}$-dependent and independent pathways in Swiss 3T3 cells. J. Biol. Chem. 262, 1159811606

Perisic O. \& Traugh J.A. (1985) Proteaseactivated kinase $\mathrm{II}$ as the mediator of epidermal growth factor-stimulated phosphorylation of ribosomal protein S6. FEBS Lett. 183, 215-218 
Pierre M., Toru-Delbauffe D., Gavaret J.M., Pomerance M. \& Jacquemin C. (1986) Activation of $S 6$ kinase activity in astrocytes by insulin, somatomedin C and TPA. FEBS Lett. 206, 162166

Rozengurt E. (1980) Cyclic AMP : a growthpromoting signal for mouse 3T3 cells. Adv. Cyclic. Nucleotide Res. 14, 429-442

Stefanovic D., Erikson E., Pike L.J. \& Maller J.L. (1986) Activation of a ribosomal S6 protein kinase in xenopus oocytes by insulin and insulinreceptor kinase. EMBO J. 5, 157-160

Tabarini D., Heinrich J. \& Rosen O.M. (1985) Purification of a bovine liver S6 kinase. Biochem. Biophys. Res. Commun. 144, 891-899
Thomas G., Siegman M. \& Gordon J. (1979) Multiple phosphorylation of ribosomal protein S6 during transition of quiescent 3T3 cells into early G1 and cellular compartmentalization of phosphate donor. Proc. Natl Acad. Sci. USA 76, 3952-3956

Toru-Delbauffe D., Gavaret J.M., Jacquemin C., Matricon C., Pomerance M. \& Pierre M. (1988) Properties of the TPA-stimulated S6 kinase from rat astroglial cells. J. Neurochem. 51, 1448-1454

Trevillyan J.M., Perisic O., Traugh J.A. \& Byus C.V. (1985) Insulin- and phorbol ester-stimulated phosphorylation of ribosomal protein S6. J. Biol. Chem. 260, 3041-3044 